\title{
Birt-Hogg-Dubé syndrome: a rare cause of familial spontaneous pneumothorax
}

\author{
HM Luk *, Tony MF Tong, Ivan FM Lo
}

Hong Kong Med J 2017;23:416.e4-5

DOI: 10.12809/hkmj165022

A family with a strong history of pneumothorax was referred to our genetic clinic for assessment. There were three siblings who had all developed spontaneous pneumothorax at the age of 30,58 , and 59 years. All were non-smokers with no preexisting pulmonary disease. High-resolution computed tomography of the thorax for all showed multiple thin-walled pulmonary cysts of variable size on both sides, mainly located at the basal and peripheral lung regions (Fig). Lung biopsy was not informative. Physical examination revealed multiple smooth, dome-shaped papules over the face and ears in one of siblings (Fig b). There were no other features of tuberous sclerosis or history of renal

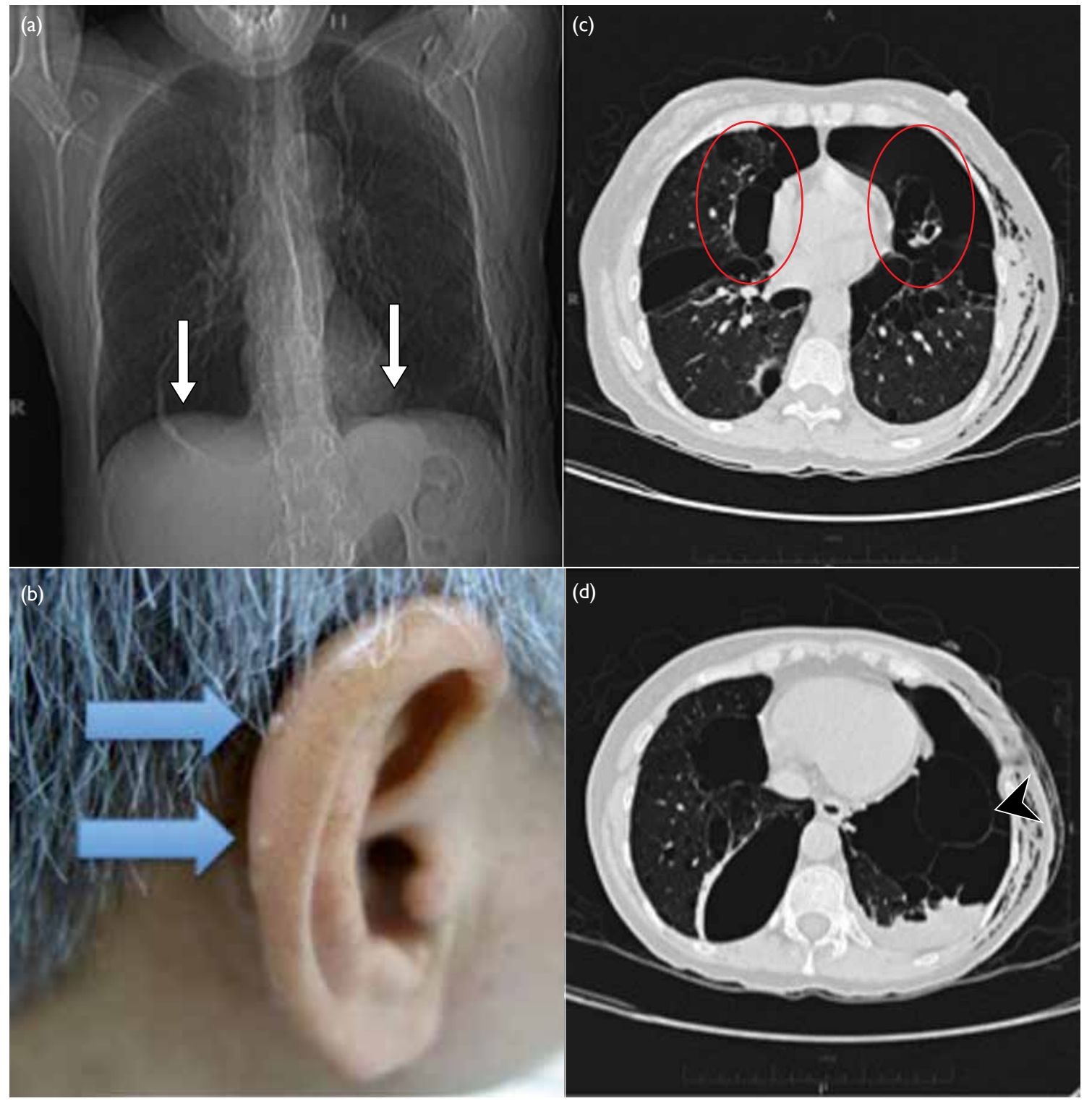

FIG. (a) Chest X-ray showing bilateral multiple pulmonary cysts over the basal region (arrows). (b) Clinical photo of fibrofolliculomas over pinna (arrows). (c and d) High-resolution computed tomography of the thorax showing lower-zone predominant pulmonary cysts of variable shapes and sizes (circles). The walls are thin, sharply demarcated and do not enhance with contrast (arrowhead) 
disease in the family. Based on the dermatological findings and diffuse multicystic lung disease, BirtHogg-Dubé (BHD) syndrome was suspected. FLCN gene analysis revealed a heterozygous FLCN\{NM_144997.5\}:c.1285dupC mutation in all affected members. The diagnosis of BHD syndrome was substantiated. Renal imaging was arranged for surveillance of potential renal cell carcinoma.

The BHD syndrome is a rare autosomal dominant disease characterised by three major organ manifestations ${ }^{1}$ :

(1) Fibrofolliculomas and other benign skin tumours such as trichodiscomas and acrochordons; these skin lesions are predominantly located on the facial, cervical, and upper truncal regions as smooth, dome-shaped, and white to fleshcoloured papules.

(2) Increased susceptibility to renal cell carcinoma of mixed histologies; the most frequent subtype is a hybrid oncocytic tumour with features of renal oncocytoma and chromophobe renal cell carcinoma.

(3) Multiple bilateral pulmonary cysts and spontaneous pneumothorax.

The clinical features of BHD syndrome are heterogeneous with wide intra-familial and interfamilial variation. It is caused by mutations of the FLCN gene. Any combination of the cutaneous, renal, and pulmonary features mentioned above present in an individual or multiple family members should alert the clinician to the possibility of BHD syndrome.

Bilateral multiple pulmonary cysts are a highly penetrant feature in BHD syndrome. As a result, the risk of pneumothorax in BHD patients is 50 times higher than that of the general population. ${ }^{2}$ Approximately $80 \%$ to $90 \%$ of BHD patients develop lung cysts, usually after early mid-adulthood. The BHD-associated lung cysts tend to be located at the basilar and mediastinal regions of the lungs, in contrast to the typically apical location in primary spontaneous pneumothorax and emphysema. Radiologically, the BHD-associated lung cysts are usually irregularly shaped, variable in size and number, and with sharply demarcated thin walls that do not enhance on computed tomographic imaging.

Fibrofolliculomas are present in more than $80 \%$ of patients with BHD syndrome and typically appear after the age of 20 years. They are domeshaped, white to flesh-coloured, non-painful and non-pruritic papules located on the facial, cervical, and upper truncal regions.

The most threatening complication of BHD syndrome is renal cell carcinoma. It occurs in approximately $15 \%$ of BHD patients by the age of 70 years. ${ }^{3}$ Therefore regular surveillance is mandatory.

Physicians should be alert to the possibility of BHD syndrome in a patient who presents with diffuse cystic lung disease, particularly in the presence of a positive family history. Early referral to a clinical genetic service and multidisciplinary management is recommended. Early diagnosis and regular renal surveillance aim to greatly reduce renal cell carcinoma-associated morbidity and mortality.

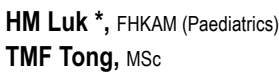

Clinical Genetic Service, Department of Health, 3/F Cheung Sha Wan

Jockey Club Clinic, 2 Kwong Lee Road, Shamshuipo, Hong Kong

* Corresponding author: luksite@gmail.com

\section{References}

1. Menko FH, van Steensel MA, Giraud S, et al. Birt-HoggDubé syndrome: diagnosis and management. Lancet Oncol 2009;10:1199-206.

2. Gupta N, Seyama K, McCormack FX. Pulmonary manifestations of Birt-Hogg-Dubé syndrome. Fam Cancer 2013;12:387-96.

3. Stamatakis L, Metwalli AR, Middelton LA, Marston Linehan W. Diagnosis and management of BHDassociated kidney cancer. Fam Cancer 2013;12:397-402. 